Onkologie 1989;12(suppl. 3):2

\title{
Impressum, Vol. 12, Supplement 3, 1989
}

\section{Anzeigen}

S. Karger Verlag für Medizin und Naturwissenschaften $\mathrm{GmbH}$, Postfach 1724, D-8034 Germering, Telefon (089) 843035.

Für den Inhalt außerhalb des redaktionellen Teiles (insbesondere Anzeigen, Industrieinformationen, Pressezitate und Kongreß-informationen) übernehmen Schriftleitung, Beirat und Verlag keine Gewähr.

Eine Markenbezeichnung kann warenzeichenrechtlich geschützt sein, auch wenn bei ihrer Verwendung in dieser Zeitschrift das Zeichen ${ }^{\circledR}$ oder ein anderer Hinweis auf etwa bestehende Schutz-rechte fehlen sollte. Für Satzfehler, insbesondere bei Dosierungs-angaben, wird keine Gewähr übernommen.

Alle Rechte, insbesondere das Recht der Vervielfältigung und Mikrokopie sowie der Übersetzung, vorbehalten. Nachdruck, auch auszugsweise, nur mit Genehmigung des Verlages. (C) Copyright 1989 by S. Karger Verlag für Medizin und Naturwissenschaften GmbH, Postfach 1724, D-8034 Germering

Satz und Druck: Walter Biering GmbH, Grafischer Betrieb, Freisinger Landstr. 21, D-8000 München 45

T1/8ymoGlanduretten

Zur additiven Therapie von Immundefekten, z.B. bei

Redaktionelle Bearbeitung und Zusammenstellung dieser Beilage: Dr. med. E. G. Coeugniet, Bad Bentheím

Wir danken nachfolgenden Firmen für ihre Unterstützung bei der Herstellung dieser Beilage: Bio-Naturkraft, Poing biosyn Arzneimittel GmbH, Stuttgart Helixor Heilmittel GmbH, Rosenfeld Midy Arzneimittel GmbH, München Dr. Kurt Mulli Nachf., Neuenburg Novipharm GmbH, Pforzheim Schaper \& Brümmer KG, Salzgitter

Infektionskrankheiten

Rheumatoider Arthritis

Immunopause

Zusammensetzung: 1 magensaft-resistentes, dünndarmlösliches Dragee enthält $80 \mathrm{mg}$ biologisch aktive Polypeptide aus Glandulae thymi sice, фstandardisiert auf Thymosin $\alpha$,).

Anwendungsgebiete: Typische Thymusmangelkrankheiten.

Gegenanzeigen und Nebenwirkungen: Bisher nicht bekannt.

Dosierung und Anwendung: 3-5 mal täglich 1 Dragee. Bei Bedarf kann diese

Dosis erhöht werden.

Darreichungsformen und Packungsgroßen: Packung mit 50 Dragées (N2)

DM 47,12. Klinikpackung mit 400 Dragées DM 250,26.

Apothekenpflichtig 


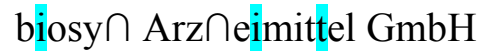

Biotechnologie im Dienste der Medizin Nobelstraße 15 — 7000 Stuttgart 80 — Tel.: 0711/6 87 4711

|1/8 1989;012:2 